\title{
Heat Pump Model for Ranque-Hilsch Vortex Tubes
}

\author{
E. J. Kolmes ${ }^{\mathrm{a}}$, V. I. Geyko ${ }^{\mathrm{a}}$, N. J. Fisch ${ }^{\mathrm{a}}$ \\ ${ }^{a}$ Department of Astrophysical Sciences, Princeton University, Princeton, New Jersey 08544, USA
}

\begin{abstract}
We describe a quantitative model for heat separation in a fluid due to motion along a pressure gradient. The physical model involved is relevant to one explanation for the temperature separation in a vortex tube. This effect has a point of saturation in which the fluid's temperature and pressure are related at its boundaries by an adiabatic law. Vortex tube models sometimes assume that this saturation is achieved in physical devices. We conclude that this is likely to be a safe assumption much of the time, but we describe circumstances in which saturation might not be achieved. We propose a test of our model of temperature separation.
\end{abstract}

Keywords: Ranque-Hilsch vortex tube, refrigeration, heat pump, thermodynamics

\section{Introduction}

A Ranque-Hilsch vortex tube is a device which separates initially constant-temperature gas into hot and cold streams. A typical vortex tube consists of a cylindrical container into which gas is injected in a direction perpendicular to the cylinder axis. The injected gas sets up a rapidly rotating flow inside the tube. Gas is allowed to exit the tube from either end, but the exits of each end are configured so that one end primarily draws gas from the outer edge of the cylinder, whereas the other end primarily lets out gas from the central region. The Ranque-Hilsch vortex tube is named for Ranque, who invented the device, and Hilsch, who made important early contributions to its study $[1,2]$. Vortex tubes are used in industry for a variety of applications, generally involving spot cooling.

Since the discovery of vortex tubes, there has been debate and discussion about the details of how the characteristic temperature separation comes about. Some early theories suggested that the effect was caused by friction between concentric annular regions of the rotating tube [2]. Deissler and Perlmutter performed an analysis [3] which suggested that turbulent shear work was the most important cause of the temperature separation. Kurosaka developed a theory [4] that explained the temperature separation in terms of acoustic streaming. Stephan et al. suggested [5] that Görtler vortices on the walls of the tube were an important factor.

One general approach, which has been used by several authors, seeks to explain the temperature separation effect in terms of adiabatic heating and cooling. The rotating flow sets up a radial pressure gradient to balance the centrifugal potential. If some fluid moves radially back and forth, it will tend to be adiabatically heated as it moves

Email address: ekolmes@princeton.edu (E. J. Kolmes) from the core to the periphery and adiabatically cooled as it moves from the periphery to the core. Kassner and Knoernschild [6] introduced the premise that radial motion will make the temperature distribution follow an adiabatic law, that is,

$$
T(r) \sim p(r)^{(\gamma-1) / \gamma} .
$$

Their explanation involved an initially irrotational vortex (that is, with angular velocity that scales with radius like $r^{-2}$ ) which converts to a rotational vortex (with constant angular velocity) due to effects at the outer boundary and the core. They suggest that the radial motion which brings about this temperature distribution will be driven by turbulence.

Ahlborn and Groves have reported experimental observations [7] of a secondary flow in a vortex tube, which includes both axial and radial motion. Their measurements did not determine whether this secondary flow was open or closed. Subsequently, Ahlborn and collaborators suggested a model in which this secondary flow played a key role $[8,9]$. In this model, the secondary flow sets up a refrigeration cycle in the vortex tube.

Computational fluid dynamics work carried out by Behera et al. [10] confirmed the existence of such a secondary flow for vortex tubes in which the cold end diameter was small compared to the diameter of the rest of the tube. However, they reported that it disappears for vortex tubes in which the cold end diameter was less small. Behera et al. found that the simulations with smaller cold end diameters more closely matched the model put forth by Ahlborn et al., but that the simulations with larger cold end diameters did not closely match that model and had substantially larger temperature separations. In general, computational studies have played an important role in vortex tube research, addressing a wide variety of issues involving vortex tube flow and temperature separation [11-15].

September 14, 2016 
Liew et al. made a quantitative model using the adiabatic law given in Eq. (1) and relying on the presence of radial motion within the vortex tube [16]. In order to predict the temperatures measured at the two exits of the tube, they also included a term to account for adiabatic deceleration as the fluid moves axially toward the hot-stream exit.

In this paper, we explore a simple analytical model of a vortex-tube-like heat pump relying on adiabatic heating and cooling of fluid moving along a pressure gradient. We will use this model to try to address some of the questions and assumptions involved in existing vortex tube models. In particular, if a subset of a fluid is moving up and down a pressure gradient, how reasonable is it to assume that the entire system will attain the temperature distribution described in Eq. (1)? Furthermore, to what extent does it matter what the radial flow looks like - that is, if the flow is due to an open secondary flow, a closed secondary flow, or a more general kind of radial mixing?

\section{The Heat Pump}

To construct a quantitative model for heat transport, suppose a parcel of gas is being moved up and down a potential gradient. A schematic of the cycle is shown in Figure 1. For simplicity, consider a gravitational potential so that the pressure is higher at smaller $z$. In practice, any pressure gradient between $z=0$ and $z=L$ produces the same effect. In a vortex tube, the centrifugal potential produces the pressure gradient.

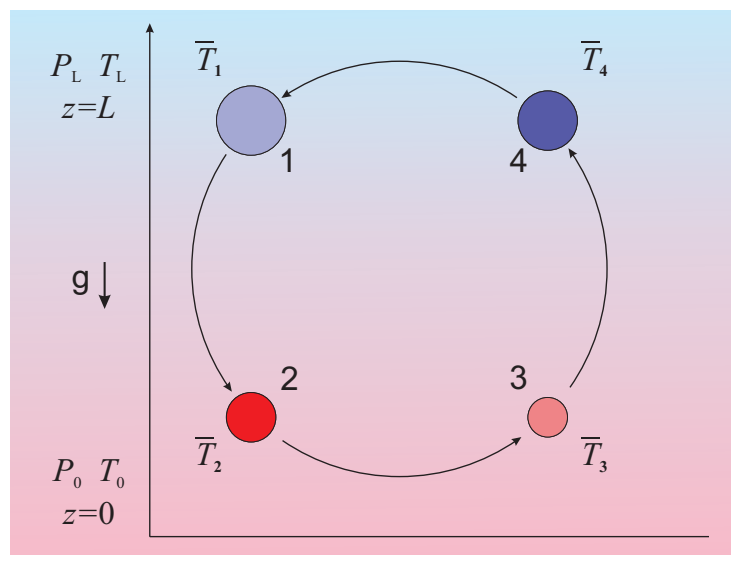

Figure 1: This diagram shows the heat transfer cycle used in our model.

Broadly speaking, our model involves parcels of gas whose motion along a pressure gradient causes them to be adiabatically cooled and heated. While they are cooled (or heated), work is done on them by the surrounding medium (or the other way around), which facilitates energy transfer between the parcel and its surroundings. However, this is not enough to establish a temperature gradient in the surrounding fluid. If the work associated with adiabatic heating and cooling were the only source of energy transfer, then the work done during the cooling step would exactly cancel with the work received in the heating step, and no net energy would be exchanged between the moving parcels and the surrounding fluid. For this reason, we include steps after each adiabatic heating or cooling step during which the moving parcel is allowed to exchange heat with its surroundings, either by conduction or by mixing.

The parcel of gas starts at some position $z=L$ and internal temperature $\bar{T}_{1}$ then moves to a new position $z=$ 0 . Suppose the background medium has some pressure distribution $p(z)$ with $p_{0} \equiv p(0)$ and $p_{L} \equiv p(L)$. Then we can define a dimensionless constant

$$
\alpha \equiv\left(\frac{p_{0}}{p_{L}}\right)^{(\gamma-1) / \gamma} .
$$

$\gamma$ denotes the specific heat ratio $c_{p} / c_{v}$. We will assume that the moving parcel is always in pressure equilibrium with its surroundings (the pressure equilibration time should be much shorter than the temperature equilibration time). If the motion of the fluid parcel is adiabatic, then the new temperature will be $\bar{T}_{2}=\alpha \bar{T}_{1}$. Then suppose the fluid element remains at $z=0$ for some period of time, during which it exchanges heat with the surrounding medium. If the surrounding medium has some temperature $T_{0}$ at $z=0$, then the temperature of the gas in the parcel during this time will satisfy the heat transfer equation

$$
\frac{d \bar{T}}{d t}=\frac{-A h}{c_{p} N_{p}}\left(\bar{T}_{2}-T_{0}\right) .
$$

Here $A$ is the area of the interface between the parcel of gas and the environment, $h$ is the heat transfer coefficient, $c_{p}$ is the constant-pressure heat capacity, and $N_{p}$ is the number of particles in the parcel. We will assume that the temperature inside the parcel is homogeneous. After heat has been exchanged for an interval $\tau_{e}$, we get a new temperature. If the heat exchange is entirely due to heat conduction, that new temperature will be

$$
\bar{T}_{3}=T_{0}+\left(\bar{T}_{2}-T_{0}\right) \exp \left(\frac{-A h \tau_{e}}{c_{p} N_{p}}\right) .
$$

If the fluid parcel then adiabatically rises back to $z=$ $L$, the new temperature will be $\bar{T}_{4}=\bar{T}_{3} / \alpha$. Finally, if the parcel once again exchanges heat isobarically with the surrounding environment before the cycle begins again, and if the background medium has temperature $T_{L}$ at $z=$ $L$, we have

$$
\bar{T}_{1}=T_{L}+\left(\bar{T}_{4}-T_{L}\right) \exp \left(\frac{-A h \tau_{e}}{c_{p} N_{p}}\right) .
$$

We will assume for the sake of simplicity that the exponential factor $-A h \tau_{e} / c_{p} N_{p}$ is the same for both points in the cycle where the parcel undergoes isobaric heat exchange (realistically, several of those terms might vary, but the overall behavior of the system should not change too 
much). We will define this factor by

$$
r \equiv \exp \left(\frac{-A h \tau_{e}}{c_{p} N_{p}}\right) .
$$

If $r=1$, then there is no transfer of energy during these steps of the cycle and the adiabatic compression and expansion steps will cancel each other out. If $r=0$, then the parcel of moving gas achieves full thermal equilibrium with its surroundings during the heat conduction steps.

If the parcel returns to the same temperature $\bar{T}_{1}$ with each cycle, then it follows that $\bar{T}_{1}, \bar{T}_{2}, \bar{T}_{3}$, and $\bar{T}_{4}$ can each be expressed in terms of the temperature of the background fluid. For instance,

$$
\bar{T}_{1}=\frac{\alpha T_{L}+r T_{0}}{\alpha(1+r)} .
$$

We can use this information to solve for the total heat transferred between the parcel and the surrounding fluid during the second and fourth steps. They are

$$
\begin{gathered}
Q_{\mathrm{bot}}=\frac{c_{v} N_{p}(1-r)}{1+r}\left(\alpha T_{L}-T_{0}\right) \\
Q_{\mathrm{top}}=-\frac{c_{v} N_{p}(1-r)}{\alpha(1+r)}\left(\alpha T_{L}-T_{0}\right) .
\end{gathered}
$$

Since total heat must be conserved and $Q_{\text {bot }}+Q_{\text {top }} \neq 0$, there must be some work, not taken into account yet, required for the compression and decompression of the parcel of gas. During an adiabatic process, the parcel's temperature satisfies

$$
\bar{T}(z+d z)=\bar{T}(z)\left(\frac{p(z+d z)}{p(z)}\right)^{(\gamma-1) / \gamma} .
$$

As such, the work deposited at a given height when the parcels moves a small (signed) distance $d z$ is given by

$$
d W=-c_{v} N_{p} \bar{T}(z) \frac{\gamma-1}{\gamma} \frac{p^{\prime}(z)}{p(z)} d z
$$

For a particle being compressed on its way from $z=L$ to $z=0$, we can write this expression in terms of the surrounding fluid temperature $T(z)$ rather than the parcel temperature $\bar{T}(z)$ by using Eq. (7) and the usual expression for temperature during adiabatic compression to get

$$
\begin{aligned}
& d W_{12}=-c_{v} N_{p} \bar{T}_{1} \frac{\gamma-1}{\gamma}\left(\frac{p(z)}{p_{L}}\right)^{(\gamma-1) / \gamma} \frac{p^{\prime}(z)}{p(z)} d z \\
& \frac{d W_{12}}{d z}=-c_{v} N_{p} \frac{\alpha T_{L}+r T_{0}}{\alpha(1+r)} \frac{d}{d z}\left(\frac{p(z)}{p_{L}}\right)^{(\gamma-1) / \gamma} .
\end{aligned}
$$

An analogous argument for the decompression as the parcel goes from $z=0$ to $z=L$ gives

$$
\frac{d W_{34}}{d z}=-c_{v} N_{p} \frac{r \alpha T_{L}+T_{0}}{1+r} \frac{d}{d z}\left(\frac{p(z)}{p_{0}}\right)^{(\gamma-1) / \gamma} .
$$

Combining Eqs 13 and 14, and keeping in mind the fact that the parcel is moving in opposite directions during the compression and decompression steps, we can compute a total profile for the change in energy of the background fluid due to the compression and decompression steps:

$$
\frac{d W}{d z}=c_{v} N_{p} \frac{1-r}{1+r}\left(\alpha T_{L}-T_{0}\right) \frac{d}{d z}\left(\frac{p(z)}{p_{0}}\right)^{(\gamma-1) / \gamma}
$$

Eqs. (8), (9), and (15) provide a complete description of the energy deposition profile due to this heat pump. The heat pump moves around energy but (so long as we don't account for whatever force is actually moving the parcel of gas back and forth) it does conserve energy:

$$
Q_{\mathrm{bot}}+Q_{\mathrm{top}}+\int_{0}^{L} \frac{d W}{d z} d z=0
$$

These calculations are for the total heat deposited at a given value of $z$ per cycle of a heat pump including $N_{p}$ circulating particles. Let $n_{p}$ be the three-dimensional volumetric density of circulating particles and let $\tau_{f}$ be the total time it takes the flow to complete one cycle from $z=L$ to $z=0$ and back. For a given one-dimensional subset of a three-dimensional system (i.e. a choice of $x$ and $y$ ), the rate of heat deposition per unit length will be given by

$$
\begin{aligned}
\dot{q}(z) & =\frac{c_{v} n_{p} L}{\tau_{f}} \frac{1-r}{1+r}\left(\alpha T_{L}-T_{0}\right) \\
& \times\left\{\frac{d}{d z}\left(\frac{p(z)}{p_{0}}\right)^{1 / c_{p}}+\delta(z)-\frac{\delta(z-L)}{\alpha}\right\} .
\end{aligned}
$$

As we have derived them, these equations describe a closed cycle in which the circulating parcels of fluid are always separate from the surrounding fluid. In a real system, the distinction between circulating fluid and background fluid might not be so clear.

We can recover the behavior of a more general flow - albeit somewhat crudely — by varying the parameter $r$. When fluid is exchanged between the parcel and the background at the top and bottom of the system, that is equivalent to a closed circulation which exchanges heat more efficiently during the isobaric steps of the cycle. In fact, for $r=0$, there is no reason why the upward-moving and downward-moving fluids have to contain any of the same material at all, so long as the net upward and downward fluxes are equal.

Note that, so far, our treatment of this model makes no assumptions about the source of the pressure gradient that compresses and decompresses the circulating parcels. To make the system intuitive, we associate lower values of $z$ with higher pressures (so that one could imagine a pressure profile resulting from a gravitational field) but the physics would work identically if we considered a centrifugal potential with high pressure at larger radial positions. 


\section{Forced Equilibrium: Analytical Solutions}

The full, time-dependent dynamics of a system with this kind of heat pump could be quite complicated. However, if instead we solve for static (forced) equilibria of the system, we can reduce the problem to three coupled differential equations:

$$
\begin{array}{cc}
p(z)=n(z) T(z) \quad & (\text { equation of state) } \\
\frac{\partial p}{\partial z}+n(z) \nabla \phi(z)=0 \quad(\text { momentum balance) } \\
\frac{\partial}{\partial z}\left(\kappa \frac{\partial T}{\partial z}\right)+\dot{q}(z)=0 \quad \text { (heat balance) }
\end{array}
$$

Here $\phi(z)$ is a potential energy (for instance, $m g z$ in a constant gravitational field). We will assume that, for some constant $\kappa_{0}$, the conductivity $\kappa$ of a gas takes the form [17]

$$
\kappa=\kappa_{0} \sqrt{T}
$$

Anywhere away from $z=0$ and $z=L$, we can make use of the fact that $\dot{q}(z)$ is a total derivative and integrate Eq. (20) to get

$$
\kappa \frac{\partial T}{\partial z}=C-\frac{c_{v} n_{p} L}{\tau_{f}} \frac{1-r}{1+r}\left(\alpha T_{L}-T_{0}\right)\left(\frac{p(z)}{p_{0}}\right)^{1 / c_{p}} .
$$

Our system will have a ceiling at $z=L$ and a floor at $z=0$. We can solve for the constant $C$ by using the boundary conditions. If the temperature at $z=0$ is at steady-state, then using Eq. (17),

$$
\left.\kappa \frac{\partial T}{\partial z}\right|_{z=0}=-\frac{c_{v} n_{p} L}{\tau_{f}} \frac{1-r}{1+r}\left(\alpha T_{L}-T_{0}\right) .
$$

To satisfy both Eq. (22) and Eq. (23), it must be true that the integration constant $C=0$. The boundary condition at $z=L$ reduces to the same constraint.

In general, the effective potential $\phi$ for a vortex tube may not be simple. The angular velocity of the flow may not be constant over the whole system [18]. As such, we will pick the simplest possible form for $\phi$ that still captures the essential behavior of the system: a linear potential $\phi=m g z$. With this choice of potential, the system turns out to be directly integrable. First, we invoke Eqs. (18) and (19) and divide both sides by $T$ :

$$
\begin{aligned}
& \frac{\kappa}{T} \frac{\partial T}{\partial z}=\frac{c_{v} n_{p} L(1-r)\left(\alpha T_{L}-T_{0}\right)}{m g \tau_{f}(1+r)} \\
& \times\left(\frac{p(z)}{p_{0}}\right)^{1 / c_{p}} \frac{p^{\prime}(z)}{p(z)} .
\end{aligned}
$$

Define the parameters $\xi$ and $\psi$ by

$$
\begin{aligned}
\xi & \equiv \frac{c_{v} c_{p} n_{p} L(1-r)}{2 \kappa_{0} m g \tau_{f}(1+r)}\left(\alpha T_{L}-T_{0}\right) \\
& \equiv \psi\left(\alpha T_{L}-T_{0}\right) .
\end{aligned}
$$

In terms of these parameters,

$$
\begin{gathered}
\frac{\partial \sqrt{T}}{\partial z}=\xi \frac{\partial}{\partial z}\left(\frac{p(z)}{p_{0}}\right)^{1 / c_{p}} \\
\Rightarrow \sqrt{T(z)}=\sqrt{T_{0}}+\xi\left(\frac{p(z)}{p_{0}}\right)^{1 / c_{p}}-\xi .
\end{gathered}
$$

Combining Eqs. (18), (19), and (28), the momentum balance equation becomes the following:

$$
\begin{aligned}
\left\{\left(\sqrt{T_{0}}-\xi\right)^{2}\right. & +2 \xi\left(\sqrt{T_{0}}-\xi\right)\left(\frac{p(z)}{p_{0}}\right)^{1 / c_{p}} \\
& \left.+\xi^{2}\left(\frac{p(z)}{p_{0}}\right)^{2 / c_{p}}\right\} \frac{p^{\prime}(z)}{p(z)}=-m g
\end{aligned}
$$

This can be integrated directly to get

$$
\begin{aligned}
\left(\sqrt{T_{0}}\right. & -\xi)^{2} \log \left(\frac{p(z)}{p_{0}}\right) \\
& +2 c_{p} \xi\left(\sqrt{T_{0}}-\xi\right)\left\{\left(\frac{p(z)}{p_{0}}\right)^{1 / c_{p}}-1\right\} \\
& +\frac{c_{p} \xi^{2}}{2}\left\{\left(\frac{p(z)}{p_{0}}\right)^{2 / c_{p}}-1\right\} \\
& =-m g z .
\end{aligned}
$$

In principle, Eqs. (28) and (30), together with energy conservation and conservation of particle number, are enough to determine the allowed equilibria. In particular, note that the pressure distribution always appears in these equations in the combination $p(z) / p_{0}$, so we can easily conserve particle number by picking $p_{0}$. The relationship between $T(z)$ and $T_{0}$ is a little more complicated, but we can get energy conservation in a similar way.

To make further sense of Eqs. (28) and (30), it is helpful to rewrite them with $z=L$ :

$$
\begin{array}{r}
\alpha\left(\sqrt{T_{0}}-\sqrt{T_{L}}\right)=\psi(\alpha-1)\left(\alpha T_{L}-T_{0}\right) \\
\left(\sqrt{T_{0}}-\xi\right)^{2} \log \alpha+\frac{2\left(\sqrt{T_{0}}-\xi\right) \xi(\alpha-1)}{\alpha} \\
+\frac{\left(\alpha^{2}-1\right) \xi^{2}}{2 \alpha^{2}}=\frac{m g L}{c_{p}} .
\end{array}
$$

These equations become somewhat simpler in terms of the following dimensionless parameters:

$$
\begin{aligned}
\Psi & \equiv \psi \sqrt{T_{0}} \\
\Xi \equiv \frac{\xi}{\sqrt{T_{0}}} & =\Psi\left(\frac{\alpha T_{L}}{T_{0}}-1\right) \\
\Gamma & \equiv \frac{m g L}{c_{p} T_{0}}
\end{aligned}
$$

Then Eqs. (31) and (32) become

$$
\frac{T_{L}}{T_{0}}=\left(\frac{-\alpha+\sqrt{\alpha^{2}+4 \Psi \alpha(\alpha-1)(\alpha-\Psi+\alpha \Psi)}}{2 \Psi \alpha(\alpha-1)}\right)^{2}
$$




$$
\begin{aligned}
(1-\Xi)^{2} \log \alpha & +\frac{2\left(\Xi-\Xi^{2}\right)(\alpha-1)}{\alpha} \\
& +\frac{\left(\alpha^{2}-1\right) \Xi^{2}}{2 \alpha^{2}}=\Gamma .
\end{aligned}
$$

Note that getting Eq. (36) did involve picking a branch of a quadratic solution; the other branch gives unphysical results in, for instance, the limit $\alpha \rightarrow 1$.

Plugging Eq. (36) into Eq. (34), and plugging that into Eq. (37), we have one equation relating $\Psi, \Gamma$, and $\alpha . \Psi$ can be thought of as a measure of the strength of the heat pump. $\Gamma$ is a normalized potential strength. $\alpha$ contains information about the size of the pressure drop across the system.

So far, this analysis has assumed that $\alpha T_{L}$ is not exactly equal to $T_{0}$. In fact, there are equilibria in which $\alpha T_{L}$ becomes arbitrarily close to $T_{0}$. However, it turns out that we cannot satisfy the heat equation in the interior of the system and at the boundaries if $\alpha T_{L}=T_{0}$ and $\alpha>1$. As such, our earlier derivations are safe.

\section{Forced Equilibrium: Numerical Solutions}

The equilibria are governed by Eqs. (36) and (37), but interpreting implicit equations of this kind can be difficult. It is useful to look at numerically-computed pictures of the surface in parameter space that is defined by these equations. Two such pictures are shown in Figure 2.

It is possible to reconstruct different aspects of the numerical solution analytically though various series solutions and limiting cases. We'll make just a few observations here. First, the edge of the solution-surface for very small $\Psi$ can be recovered by taking the limit as $\Psi \rightarrow 0$ in Eqs. (36) and (37). In this limit, $T(z)$ becomes constant and Eq. (37) yields $\alpha=e^{\Gamma}$, which is equivalent to $p_{L}=p_{0} e^{-m g L / T_{0}}$. This is the simple solution that we would expect without any heat pump, and we can easily see the corresponding exponential curve on the right-hand side of the surface.

Another notable feature of the surface is the vertical structure roughly parallel to the $\alpha-\Psi$ plane. Naïvely, for a fixed $\Psi$ one might expect that it should always be possible to increase the strength of the potential and get out a larger pressure gradient (that is, increase $\Gamma$ and see an equilibrium with a larger value of $\alpha$ ). Instead, there appears to be an upper limit for any given $\Psi$ on how large $\Gamma$ is allowed to be, and the large- $\alpha$ solutions form this vertical structure instead of corresponding to very large values of $\Gamma$. Furthermore, there are some choices of $\Gamma$ and $\Psi$ (always with $\Gamma>0.5$ ) for which there appear to be two allowed values of $\alpha$ that produce equilibrium.

A full stability analysis of this system, taking into account the reaction of the system to any possible perturbation of the density, temperature, and velocity profiles, is beyond the scope of this paper. However, in the regions where there are two possible equilibria with the same $\Gamma$ and $\Psi$, there are reasons to believe that one equilibrium is stable and the other is unstable.

The time-dependent dynamics of an ideal fluid with heat conduction (but, for the sake of simplicity, without any viscous effects) are given by [19]

$$
\begin{gathered}
\frac{\partial n}{\partial t}+\frac{\partial(n u)}{\partial z}=0 \\
m n\left(\frac{\partial u}{\partial t}+u \frac{\partial u}{\partial z}\right)+m n g+\frac{\partial(n T)}{\partial z}=0 \\
\frac{\partial}{\partial t}\left(\frac{n m u^{2}}{2}+c_{v} n T\right) \\
+\frac{\partial}{\partial z}\left[n u\left(\frac{m u^{2}}{2}+c_{p} T\right)-\kappa \frac{\partial T}{\partial z}\right]=\dot{q} .
\end{gathered}
$$

Here $u(z)$ is the fluid velocity at $z$.

Consider the system's response to a small perturbation in the gravitational parameter $g$ and the heat pump parameter $n_{p}$, so that $g \rightarrow g+\delta g$ and $n_{p} \rightarrow n_{p}+n_{p} \delta g / g$. Then, as per Eqs. (25) and (35), $\Gamma$ will be perturbed but $\Psi$ will remain unchanged.

If the system was initially in equilibrium, and if $\delta g>0$, then the initial effect of the perturbation will be to make $\partial u / \partial t<0$ for all $z, \partial p / \partial t>0$ for $z=0$, and $\partial p / \partial t<0$ for $z \neq 0$. The effect on $\partial p / \partial t$ comes from the relationship between $n_{p}$ and $\dot{q}$; see Eq. (17). Once $u(z)<0$, we can see from Eqs. (38) and (40) that the density and pressure will increase at the bottom of the system and decrease at the top, at least initially. On the other hand, if $\delta g<0$, the initial effect of the perturbation will be reversed: $\partial u / \partial t$ will be positive for all $z$ and the perturbation to $n_{p}$ will decrease $\partial p / \partial t$ at $z=0$ but increase it for all other $z$. Therefore, if we perform this perturbation, then we know that $\alpha$ will initially increase if $\delta g>0$ and decrease if $\delta g<$ 0 .

Of course, we aren't primarily interested in the response of the system to perturbations in $g$ or $n_{p}$. For the purposes of understanding stability, we mostly care about perturbations in the profiles $u(z), n(z)$, and $T(z)$. But consider the equilibrium profiles for some choice of $\Gamma$ and $\Psi$ as an infinitesimal perturbation of the equilibrium profiles for an otherwise identical system in which $g$ and $n_{p}$ differ by $\delta g$ and $n_{p} \delta g / g$, respectively.

Looking at the shape of the surfaces shown in Figure 2, we can see that the perturbation $g \rightarrow g+\delta g$ and $n_{p} \rightarrow$ $n_{p}+n_{p} \delta g / g$ is equivalent to a perturbation that makes $\alpha$ either a little smaller or a little larger. Because of the shape of the surface, a positive $\delta g$ will be associated with a negative $\delta \alpha$ wherever there is a single solution and on the smaller- $\alpha$ branch in the region where there are multiple solutions. A negative $\delta g$ will be associated with a positive $\delta \alpha$ on the larger- $\alpha$ branch of the multiple-valued part of the surface.

So, at least for this very specific class of perturbation, $\dot{\alpha}$ and $\delta \alpha$ will initially have opposite signs on the lower part of the surface and the same signs on the upper part of the surface. This suggests that the upper branch, where the 

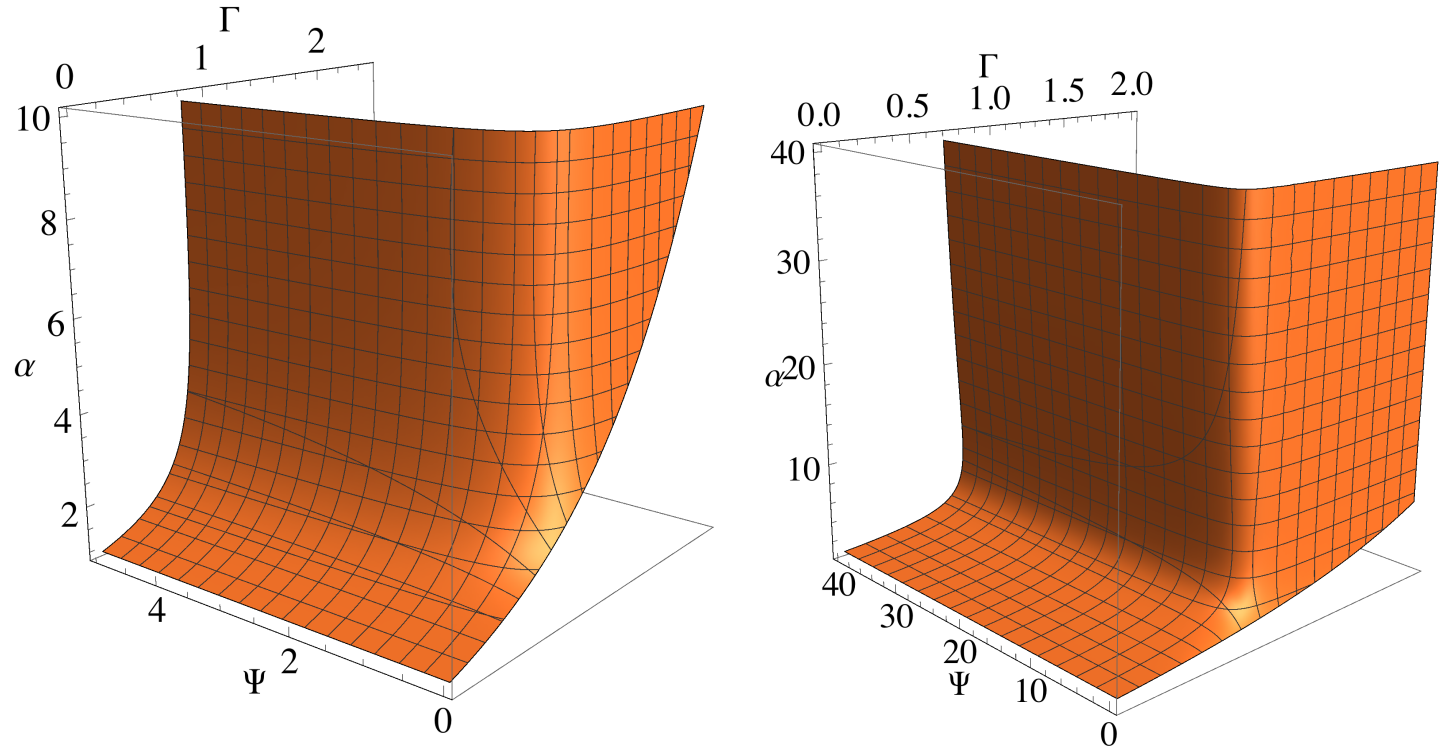

Figure 2: These images show the same numerically generated equilibrium surface in terms of $(\Psi, \Gamma, \alpha)$ coordinates. The plot on the right shows a larger range of parameter values.

surface curves back on itself, is unstable, whereas the lower branch is stable. However, it is not a rigorous proof, both because we have only described the response of the system for small times and because we have discussed the response of the system only to a specific class of perturbations.

This behavior is interesting, but it should not be overemphasized. As we will see in Section 5, the regimes in which the equilibrium $\alpha$ is not a single-valued function of $\Psi$ and $\Gamma$ are almost certainly not relevant to the behavior of actual vortex tubes. Multiple-valued equilibria don't start showing up until $\Gamma>0.5$, and realistic values for $\Gamma$ in a vortex tube are about an order of magnitude smaller. Furthermore, recall that our heat pump model involves parcels of fluid moving up to $z=L$ and exchanging heat with the surrounding medium there. If the density of particles at the top of the system is depleted, there are physical reasons to think that our model should break down. As such, we should be careful about our model's predictions when $\alpha$ is large.

\section{Predicting Temperature Separation}

Once we have some understanding of what the equilibrium surface looks like, we can move to the more practical problem: predicting the size of the temperature separation due to this kind of heat pump. The ratio of the boundary temperatures $T_{L} / T_{0}$ is specified by $\alpha$ and $\Psi$. When $T_{L} / T_{0} \rightarrow 1 / \alpha$, the heat pump turns off (or "saturates"). A surface showing the boundary temperature ratio $T_{L} / T_{0}$ as a function of $\Psi$ and $\alpha$ is shown in Figure 3, along with the reference surface $T_{L} / T_{0}=1 / \alpha$.

So, what kind of parameters should we be thinking about if we want to understand the behavior of a physical vortex tube? Vortex tubes can vary substantially in their geometry and their performance $[11,14,15]$, but we would like at least some very rough estimates. $\alpha$ is relatively straightforward to estimate, since it follows directly from measurements of the radial pressure profile. Experimental studies [20,21] suggest that we should expect $\alpha$ somewhere around 1.1, if we use static rather than total pressures.

Getting a good estimate for $\Gamma$ is a little more complicated. In a physical vortex tube, an effective centrifugal potential $m v_{\theta}^{2} / 2$ takes the place of the gravitational potential $m g z$ with which we originally defined $\Gamma$. We should take $\Gamma$ to be the difference in potential energy between the two boundaries of the system (the core and the outer radial edge), divided by $c_{p} T_{0}$. Therefore, estimating $\Gamma$ requires the rotational velocity $v_{\theta}$ at the edge of a typical vortex tube. Experimental studies [18, 20, 21] suggest that a typical velocity might be on the order of $150 \mathrm{~m} / \mathrm{s}$. For a vortex tube filled with nitrogen, that would suggest $\Gamma \sim 0.035$.

Perhaps most interesting is the parameter $\Psi$. According to the numerical results presented in Figure $3, T_{L} / T_{0}$ converges to $1 / \alpha$ once $\Psi$ is greater than 10 or 20 . If we make the substitution $m g L \rightarrow m v_{\theta}^{2} / 2$, then $\Psi$ becomes

$$
\Psi=\frac{c_{v} c_{p} n_{p} L^{2} \sqrt{T_{0}}}{\kappa_{0} m v_{\theta}^{2} \tau_{f}} \frac{1-r}{1+r} .
$$

In the relevant temperature ranges, nitrogen should have a thermal conductivity of about $2 \times 10^{21} \mathrm{~m}^{-1} \mathrm{~s}^{-1}[22]$. We'll consider a tube with $L \sim 10 \mathrm{~cm}$. Experimentally measured radial velocities [18] have been on the order of $15 \mathrm{~m} / \mathrm{s}$. If we suppose that a secondary circulation is roughly circular with a diameter of $L$ and a mean velocity of $15 \mathrm{~m} / \mathrm{s}$, then $\tau_{f} \sim 0.02 \mathrm{~s}$.

Getting a universally accurate estimate for $n_{p}$, the density of circulating particles, is difficult. Ahlborn and Groves, in their experimental report of a secondary flow, estimated 

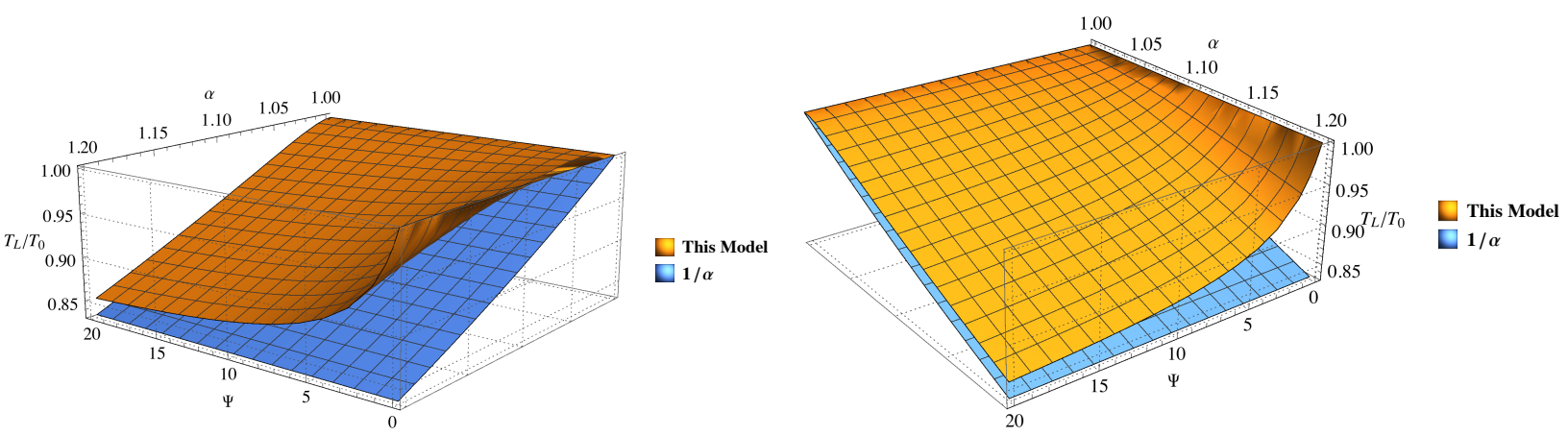

Figure 3: This surface (displayed from two different angles) shows the dependence of the temperature on $\Psi$ and $\alpha$. Note that the parameter $\Gamma$ is uniquely specified by $\alpha$ and $\Psi$, so it is not an independent parameter in these plots.

that the flow of mass per second in the secondary circulation was within about a factor of two of the mass flow in the primary circulation [7]. From pressure measurements, we can estimate that the density of the gas in a vortex tube is within an order of magnitude of atmospheric pressure, so we can estimate that $n_{p}$ is about the number density of gas at sea level, that is, around $2.7 \times 10^{25} \mathrm{~m}^{-3}$. Then, if $T_{0} \sim 320$ degrees Kelvin and $v_{\theta} \sim 150 \mathrm{~m} / \mathrm{s}$, we find that

$$
\Psi \sim 10^{5} \frac{1-r}{1+r} .
$$

Several of the numbers that went into that estimate were very rough. However, $10^{5}$ is a large number. If $r$ is not very, very close to 1 , then the estimate for $\Psi$ could be quite far off without changing the fact that we should expect the heat pump to have reached saturation.

What happens, then, if $r \approx 1$ ? A Taylor expansion of Eq. (6) gives that

$$
\Psi \approx \frac{c_{v} n_{p} L^{2} T_{0} A h \tau_{e}}{2 \kappa m v_{\theta}^{2} N_{p} \tau_{f}}
$$

If $\Delta x$ is a characteristic thickness of the boundary across which the temperature transitions between the temperature of the parcel and the temperature of its surroundings, then the heat transfer coefficient $h$ is $\kappa / \Delta x$. In that case,

$$
\Psi \approx \frac{c_{v} L^{2}}{2 \Delta x} \frac{T_{0}}{m v_{\theta}^{2}} \frac{A}{V} \frac{\tau_{e}}{\tau_{f}} .
$$

If $\ell$ is a characteristic length of the system and $V$ is the volume of a typical circulating parcel, then $A / V \sim 1 / \ell$. $\Delta x$ is probably on the same order as $\ell$. The heat exchange time $\tau_{e}$ should not exceed half of the flow cycle time $\tau_{f}$, but they might be on the same order. In that case,

$$
\Psi \approx \frac{L^{2}}{\ell^{2}} \frac{c_{v} T_{0}}{2 m v_{\theta}^{2}} \frac{\tau_{e}}{\tau_{f}} .
$$

In a typical vortex tube, $c_{v} T_{0} / 2 m v_{\theta}^{2}$ is about $10 . L^{2} / \ell^{2}$ will be greater than one and $\tau_{e} / \tau_{f}$ will be less than one. In a system that relies entirely on conduction for heat transfer, it seems very plausible that the heat pump might reach saturation, but not necessarily a forgone conclusion.
Be that as it may, if even a few percent of the parcel (or secondary flow) is exchanged with fluid from the surrounding medium at each end during each iteration of the cycle, then $r$ will be much larger and the system will saturate. The flow of fluid up and down the pressure gradient has to be completely closed if the system is going to be anywhere other than the saturation limit.

\section{Discussion}

Our model includes a number of potentially important simplifications. First, it is a one-dimensional model which does not include any axial effects. The assumption that the essence of the Ranque-Hilsch temperature separation effect can be captured without any axial effects is not an unambiguously safe one. For example, the refrigeration cycle model presented by Ahlborn et al. involved a secondary flow that moved axially as well as radially [8, 9], although that model and the one explored here have a number of qualitative similarities.

The model presented by Liew et al. was largely onedimensional, and in fact their predicted temperature separation between the core and the radial outer edge is identical to our result in the saturated-heat-pump limit [16]. However, Liew et al. include an additional term to account for adiabatic deceleration of the fluid at the edge of the cylinder as it moves toward the hot exit of the tube. Our model describes the radial temperature separation rather than the difference between the hot-end and cold-end exit temperatures. A term of the kind used by Liew et al. might be necessary in order to translate from one result to the other.

Furthermore, the results from Sections 3 and 4 are for a radial slice of the system that is in equilibrium. This may or may not be a good description of a real vortex tube, especially if the tube is relatively short and the axial flow rate is relatively large. Liew et al. have suggested [16] that overly short vortex tubes may have worse performance, essentially for this reason.

We should also keep in mind that although much of the physics involved turns out the same way for any choice of potential, our choice to assume a gravitational potential 
(or, equivalently, a rotation profile with $\omega \sim r^{-1 / 2}$ ) does have some real consequences. For instance, the pressure equilibrium equations would turn out somewhat differently for different potentials. In fact, the rough estimates in the previous section suggest that the experimentally observed values of $\Gamma$ are smaller than Eq. (37) would predict for the estimated values of $\alpha$. Our assumption of a linear potential may explain this.

Despite the simplifications involved in this model, it may provide some insights into what factors should affect the performance of a vortex tube. Once the heat transport has saturated, then for a given radial slice of the tube, the problem of optimizing the radial temperature separation reduces to the maximization of $\alpha$. One way of increasing $\alpha$ would be to increase the radial pressure gradient, which could be accomplished by increasing the angular velocity of the spinning gas.

The parameter $\alpha$ also depends on the specific heat capacity $c_{p}$. Smaller specific heat capacities result in larger values of $\alpha$. In particular, at saturation, and to first order in $(\alpha-1)$, the equilibria given by Eqs. (36) and (37) become

$$
\begin{aligned}
\alpha & =1+\Gamma \\
\frac{T_{L}}{T_{0}} & =\frac{1}{1+\Gamma} .
\end{aligned}
$$

Recall that $\Gamma \propto 1 / c_{p}$. One might expect that switching between gases could improve performance, especially between diatomic and monatomic gases [23]. However, this is contingent on the rotational energy $m v_{\theta}^{2} / 2$ at the edge remaining constant. Some experimental work has already been done concerning the behavior of different gases and mixtures of gases in vortex tubes [24-26], but they have not fully resolved whether or not this prediction of our model is correct.

Of course, if the heat transport were not saturated, then this becomes somewhat more complicated. Switching between gases could modify the flow inside the vortex tube in such a way that the parameter $\Psi$ could change and the system could be pushed toward or away from saturation. Our model does not predict the dependence of $r, \tau_{e}$, and $\tau_{f}$ on factors like the molecular mass of the gas in the tube. These dependences are also somewhat more complicated if we want to predict measurements of the hot and cold exit temperatures rather than predicting the radial temperature profile at a particular axial location along the tube (the stagnation terms will have their own dependences on the adiabatic index $\gamma$ and the rotational velocity).

The physics that we describe could potentially be useful for a variety of applications outside of the world of vortex tubes. For instance, one might look for astrophysical applications anywhere there is a fluid with a temperature gradient that is stable to the convective instability but that still contains some kind of mixing or other motion along a pressure gradient. In fact, in the context of rotating astrophysical disks, the "backwards transport" discussed by Balbus [27] has a great deal in common with the Ranque-Hilsch temperature separation effect. There are also similarities between our model and the physics of thermoacoustic heat pumps. These devices use acoustic waves to compress and decompress parcels of gas in order to move heat up a temperature gradient. One type of thermoacoustic heat pump can be described as a Brayton cycle [28], much like our model of Ranque-Hilsch temperature separation.

Ranque-Hilsch temperature separation is not the only kind of interesting heat transport effect that can happen in a rotating gas. For instance, for a quickly compressed rotating gas, the piezo-thermal effect [29] could set up a temperature gradient such that the core is heated and the periphery is cooled (it could cool the core and heat the periphery if a rotating column of gas were decompressed instead). It would be interesting to see experimental and theoretical investigations into the behavior of a vortex tube that is compressed or decompressed during operation, given the variety of additional physics that might come into play [30, 31].

\section{What Can Be Done to Test This Model?}

In order to validate this model of Ranque-Hilsch temperature separation, especially as opposed to the saturationregime temperature profile described by others $[6,16]$, it would be useful to make experimental measurements of the temperature separation for a vortex tube operating outside of the saturation regime. Ideally, we would want $\Psi$ (and, to a lesser extent, $\alpha$ ) to be as small as possible while still achieving a measurable temperature separation. This might be achieved by producing relatively low angular velocities in the tube and radial motion that relies as much as possible on a closed secondary flow, with minimal turbulence.

One potential difficulty in validating this model comes from the fact that it describes radial heat transport but does not consider effects due to axial flow. It would be interesting to see the behavior of a device with radial flow that mimics what is found in a vortex tube (likely driven mechanically) but with sealed ends and no axial flow. If such a device were to achieve temperature separation similar to what appears in a vortex tube (perhaps up to a stagnation term), that would suggest that the heat transfer can correctly be described in terms of the radial behavior of the fluid in a given slice of the device.

\section{Conclusions}

One promising possible explanation for the vortex tube heat separation effect involves a heat pump driven by radial motion. In this paper, we have presented a simple analytical model intended to capture the basic behavior of such a heat pump, and to explore which parameters of the 
vortex tube flow are likely to be most important to the heat separation effect.

Our model suggests that, under many circumstances, the heat pump effect may "saturate" in such a way that driving a more vigorous secondary flow (or turbulence, or whatever is causing fluid to move radially back and forth within the tube) will not change the magnitude of the temperature separation. In this case, the temperature separation reduces to a simple relation involving the pressures at the boundaries. This saturation appears to be plausible.

However, this saturation is not inevitable. Depending on the geometry of the flow, and on the quantity of radially-moving fluid, a vortex tube might not reach saturation, in which case the temperature separation will take a substantially more complicated form. The most important factor for a vortex tube not to reach saturation is that the heat exchange between circulating fluid and background fluid be entirely conductive; if even a few percent of the circulating fluid mixes with the background on each cycle, then saturation is almost certain. Although the useful applications of the vortex tube effect are likely in the regime of saturation, it is precisely when saturation is not achieved that the details of the physical processes underlying the model equations developed here may be most easily explored experimentally, thereby allowing a systematic evaluation of the model proposed here.

\section{Acknowledgments}

The authors would like to thank Dr. J.-M. Rax for his suggestions regarding thermoacoustic engines. This work was supported by the NNSA SSAA Program under Grant No. DENA0002948 and by NSF Contract No. PHY-1506122.

[1] G. Ranque, J. Phys. Rad. 7 (4), 112 (1933).

[2] R. Hilsch, Rev. Sci. Instrum. 18, 108 (1947).

[3] R. G. Deissler and M. Perlmutter, Int. J. Heat Mass Transfer 1, 173 (1960).

[4] M. Kurosaka, J. Fluid Mech. 124, 139 (1982).

[5] K. Stephan, S. Lin, M. Durst, F. Huang, and D. Seher, Int. J. Heat Mass Transfer 26, 341 (1983).

[6] R. Kassner and E. Knoernschild, Friction Laws and Energy Transfer in Circular Flow, Tech. Rep. F-TR-2198-ND (WrightPatterson Air Force Base, 1948).

[7] B. Ahlborn and S. Groves, Fluid Dyn. Research 21, 73 (1997).

[8] B. K. Ahlborn, J. U. Keller, and E. Rebhan, J. Non-Equilib. Thermodyn. 23, 159 (1998).

[9] B. K. Ahlborn and J. M. Gordon, J. Appl. Phys. 88, 3645 (2000).

[10] U. Behera, P. J. Paul, S. Kasthurirengan, R. Karunanithi, S. N. Ram, K. Dinesh, and S. Jacob, Int. J. Heat Mass Transfer 48, 1961 (2005).

[11] S. Eiamsa-ard and P. Promvonge, Renew. Sustainable Energy Rev. 12, 1822 (2008).

[12] A. Secchiaroli, R. Ricci, S. Montelpare, and V. D'Alessandro, Int. J. Heat Mass Transfer 52, 5496 (2009).

[13] H. A. Kandil and S. T. Abdelghany, Energy 84, 207 (2015).

[14] S. Subudhi and M. Sen, Renew. Sustainable Energy Rev. 52, 172 (2015).

[15] H. R. Thakare, A. Monde, and A. D. Parekh, Renew. Sustainable Energy Rev. 52, 1043 (2015).

[16] R. Liew, J. C. H. Zeegers, J. G. M. Kuerten, and W. R. Michalek, Phys. Rev. Lett. 109, 054503 (2012).
[17] E. M. Lifshitz and L. P. Pitaevskii, Physical Kinetics, 1st ed., Course of Theoretical Physics (Pergamon Press, 1981).

[18] R. Liew, J. C. H. Zeegers, J. G. M. Kuerten, and W. R. Michalek, Exp. Fluids 54, 1416 (2013).

[19] L. D. Landau and E. M. Lifshitz, Fluid Mechanics, 2nd ed., Course of Theoretical Physics (Pergamon Press, 1987).

[20] H. H. Bruun, J. Mech. Eng. Sci. 11, 567 (1969).

[21] C. M. Gao, K. J. Bosschaart, J. C. H. Zeegers, and A. T. A. M. de Waele, Cryogenics 45, 173 (2005).

[22] K. Stephan, R. Krauss, and A. Laesecke, J. Phys. Chem. Ref. Data 16, 993 (1987).

[23] C. Kittel and H. Kroemer, Thermal Physics, 2nd ed. (W. H. Freeman and Company, 1980).

[24] C. U. Linderstrøm-Lang, Int. J. Heat Mass Transfer 7, 1195 (1964).

[25] J. Marshall, Int. J. Heat Mass Transfer 20, 227 (1977).

[26] O. Aydın and M. Baki, Energy 31, 2763 (2006).

[27] S. A. Balbus, Astrophys. J. 534, 420 (2000).

[28] G. W. Swift, J. Acoust. Soc. Am. 84, 1145 (1988).

[29] V. I. Geyko and N. J. Fisch, arXiv:1604.00063.

[30] V. I. Geyko and N. J. Fisch, Phys. Rev. Lett. 110, 150604 (2013).

[31] S. Davidovits and N. J. Fisch, Phys. Rev. Lett. 116, 105004 (2016). 